\title{
High-sensitivity damage detection based on enhanced nonlinear dynamics*
}

\author{
Bogdan I Epureanu ${ }^{1,3}$, Shih-Hsun Yin ${ }^{1}$ and Mark M Derriso ${ }^{2}$ \\ ${ }^{1}$ Department of Mechanical Engineering, University of Michigan, Ann Arbor, \\ MI 48109-2125, USA \\ ${ }^{2}$ Lead, Structural Health Monitoring AFRL/VASM, Wright-Patterson AFB, OH 45433-7531, \\ USA \\ E-mail: epureanu@umich.edu
}

Received 7 April 2004, in final form 3 September 2004

Published 28 January 2005

Online at stacks.iop.org/SMS/14/321

\begin{abstract}
One of the most important aspects of detecting damage in the framework of structural health monitoring is increasing the sensitivity of the monitored feature to the presence, location, and extent of damage. Distinct from previous techniques of obtaining information about the monitored structure- such as measuring frequency response functions-the approach proposed herein is based on an active interrogation of the system. This interrogation approach allows for the embedding of the monitored system within a larger system by means of a nonlinear feedback excitation. The dynamics of the larger system is then analyzed in state space, and the shape of the attractor of its dynamics is used as a complex geometric feature which is very sensitive to damage. The proposed approach is implemented for monitoring the structural integrity of a panel forced by transverse loads and undergoing limit cycle oscillations and chaos. The nonlinear von Karman plate theory is used to obtain a model for the panel combined with a nonlinear feedback excitation. The presence of damage is modeled as loss of stiffness of various levels in a portion of the plate at various locations. The sensitivity of the proposed approach to parametric changes is shown to be an effective tool in detecting damages.
\end{abstract}

\section{Introduction}

Structural health monitoring is a pervasive need in air, space, sea and land structures [6], and may be applied online (during normal operation of the monitored system) or offline (e.g. during maintenance cycles). Online is generally preferred to offline monitoring in most applications (e.g. monitoring air-frames, unmanned air vehicles, the joint strike fighter, buildings and bridges). However, technical difficulties faced by current methods (especially for detecting incipient damage of small degree) and problems associated with taking measurements during normal operating conditions have limited the available techniques to mostly offline detection of large damage.

\footnotetext{
* An earlier version was presented at the SPIE 11th International Symposium on Smart Structures and Materials.

3 Author to whom any correspondence should be addressed.
}

Damage is detected by monitoring a feature of the system. Changes in this feature indicate damage [9]. The types of features used vary depending on the monitored system (vibration response, electromagnetic properties, ultrasound etc). A class of techniques used for damage detection are based on vibratory responses. Most such methods monitor changes in the frequencies and modes of vibration $[6,9,15,16,20]$. Other current vibration-based techniques use subspace identification and updating $[1,10,17,24,29]$, wavelet analyses [3, 22], evolutionary algorithms [30], Ritz [28] or stochastic methods [19] and others [23]. These techniques are designed for linear vibrations while far fewer apply to nonlinear systems. Some nonlinear methods are based on system identification [2, 13, 21], while others use neural networks [4, 27], phase space reconstruction [5] or Lyapunov exponents $[18,25]$. These nonlinear methods have several limitations, e.g. some have difficulty tackling highdimensional systems, others do not predict the damage location 


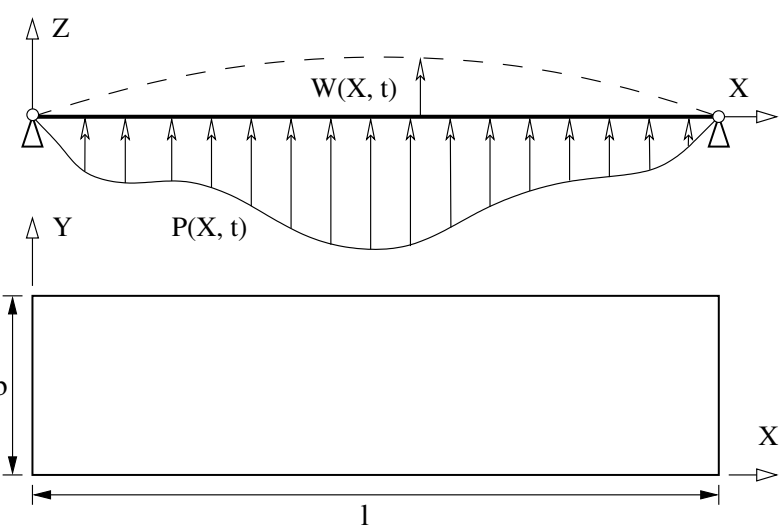

Figure 1. A panel forced by a transverse distributed force.

or level, or do not detect simultaneous damages. However, our results show that the use of nonlinearities holds a great potential. We have observed that the changes in the shape of attractors of the dynamics are large for nonlinear systems and may be used for detecting multiple damages.

Distinct from previous techniques of obtaining information about the monitored structure, an active interrogation of the system is what we propose to use. This interrogation approach allows for the embedding of the monitored system within a larger system by means of a nonlinear feedback excitation. The attractor of the dynamics of the larger system is then analyzed in state space. The shape of this attractor is used as a complex geometric feature which is very sensitive to damage. The proposed approach is implemented for monitoring the structural integrity of a panel forced by transverse loads. The proposed approach is demonstrated numerically herein. Experimental investigations involving the active interrogation proposed (by means of nonlinear feedback excitation) are feasible also, and they are expected to confirm the numerical observations.

\section{Modeling}

A panel subjected to loading is depicted in figure 1 . The structural nonlinearity is due to the coupling between stretching and bending of the panel for deflections of the order of magnitude of the panel thickness. Energy dissipation of the system is also considered by introducing internal and external damping. Finally, the damage in the panel is modeled as a local reduction in the bending stiffness of the panel (which may occur in many damage scenarios, such as the cases where yielding occurs or a crack propagates in the material).

The panel shown in figure 1 is modeled as a onedimensional, homogeneous, isotropic and elastic thin plate with pinned-pinned end points. The thickness $h$ is considered much smaller than the length $l$. Also, the width $b$ is considered much larger than the length $l$. Damping is modeled as being due to two components: external damping due to the vibration of the panel in air; and internal damping due to the viscosity of the material of the panel. To model the structural nonlinearity caused by the in-plane stretching of the panel due to out-ofplane deflections, von Karman's strain-displacement relation is used. The governing equation for the panel dynamics is obtained as

$$
D W^{\prime \prime \prime \prime}+\rho h \ddot{W}+C_{\mathrm{i}} \dot{W}^{\prime \prime \prime \prime}+C_{\mathrm{e}} \dot{W}-N W^{\prime \prime}-P(X, t)=0
$$

where $W^{\prime}$ and $\dot{W}$ represent the spatial and time derivatives of the panel deflection $W, D$ is a coefficient characterizing the bending stiffness of the panel given by $D=E h^{3} / 12\left(1-v^{2}\right)$, $C_{\mathrm{e}}$ and $C_{\mathrm{i}}$ are external and internal damping parameters, while $\rho$ is the mass density and $P(X, t)$ is the transverse distributed loading. $N$ is the in-plane tension load (assumed to be uniform along the entire length of the panel) and is expressed as follows:

$$
N=E h\left[\eta_{0}+\frac{1}{l} \int_{0}^{l} \frac{1}{2} W^{\prime 2}(\xi) \mathrm{d} \xi\right]
$$

where the first term $\left(\eta_{0}\right)$ is the initial axial strain, and the second term is the stretching of the panel due to bending. Substituting equation (2) into (1), one obtains

$$
\begin{aligned}
& D W^{\prime \prime \prime \prime}+\rho h \ddot{W}+C_{\mathrm{i}} \dot{W}^{\prime \prime \prime \prime}+C_{\mathrm{e}} \dot{W}-E h \eta_{0} W^{\prime \prime} \\
& \quad-\frac{E h}{2 l}\left[\int_{0}^{l} W^{\prime 2}(\xi) \mathrm{d} \xi\right] W^{\prime \prime}-P(X, t)=0 .
\end{aligned}
$$

The boundary conditions corresponding to a pinned-pinned panel may be expressed as

$$
\begin{gathered}
W(X=0, t)=0, \\
D W^{\prime \prime}(X=0, t)+C_{\mathrm{i}} \dot{W}^{\prime \prime}(X=0, t)=0, \\
W(X=l, t)=0, \\
D W^{\prime \prime}(X=l, t)+C_{\mathrm{i}} \dot{W}^{\prime \prime}(X=0, t)=0 .
\end{gathered}
$$

Nondimensional variables $x, w$ and $\tau$ are defined as $x=X / l$, $w=W / h$ and $\tau=t / \sqrt{\rho h l^{4} / D}$. Next, equation (3) can be nondimensionalized to obtain

$$
\begin{aligned}
w^{\prime \prime \prime \prime} & +\ddot{w}+C_{\mathrm{I}} \dot{w}^{\prime \prime \prime \prime}+C_{\mathrm{E}} \dot{w}-R_{x} w^{\prime \prime} \\
& -S\left[\int_{0}^{1} w^{\prime 2}(\xi) \mathrm{d} \xi\right] w^{\prime \prime}-F(x, \tau)=0
\end{aligned}
$$

where $w^{\prime}=\partial w / \partial x, \dot{w}=\partial w / \partial \tau$. The corresponding nondimensional coefficients in equation (4) are as follows:

- internal damping $C_{\mathrm{I}}=C_{\mathrm{i}} / \sqrt{\rho h l^{4} D}$,

- external damping $C_{\mathrm{E}}=C_{\mathrm{e}} l^{2} / \sqrt{\rho h D}$,

- in-plane pre-load $R_{x}=E h \eta_{0} l^{2} / D$,

- bending-stretching coefficient $S=E h^{3} /(2 D)=6(1-$ $v^{2}$ ) and

- input force $F(x, \tau)=l^{4} /(h D) \cdot P(x, \tau)$.

The boundary conditions can also be rewritten in nondimensional form as

$w(x=0, \tau)=0, \quad w^{\prime \prime}(x=0, \tau)+C_{\mathrm{I}} \dot{w}^{\prime \prime}(x=0, \tau)=0$,

$w(x=1, \tau)=0, \quad w^{\prime \prime}(x=1, \tau)+C_{\mathrm{I}} \dot{w}^{\prime \prime}(x=1, \tau)=0$.

The bending stiffness and Young's modulus for the damaged regions of the panel are denoted by $\bar{D}$ and $\bar{E}$. Similar to 


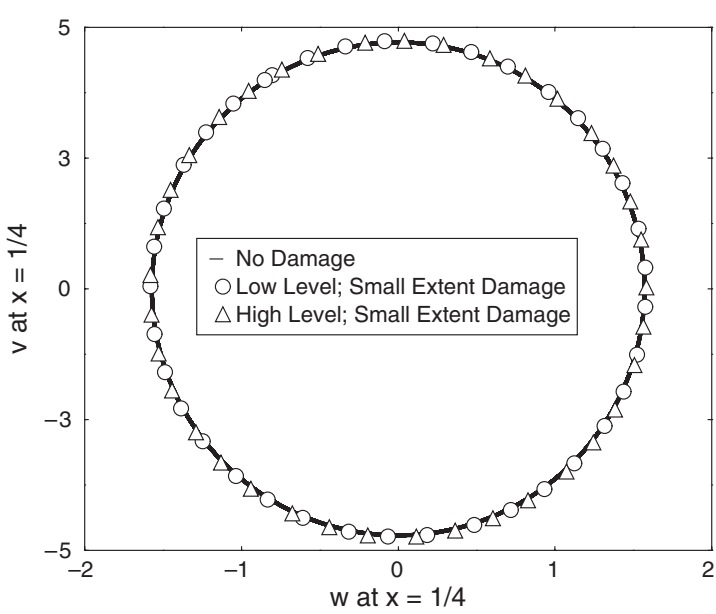

Figure 2. Quarter point dynamics ( $v=\dot{w}$ versus $w$ ) for panels with different levels of damage and subjected to a harmonic excitation force at their middle.

equation (3), the governing equation of the damaged regions of the panel may be expressed as

$$
\begin{aligned}
& \bar{D} W^{\prime \prime \prime \prime}+\rho h \ddot{W}+C_{\mathrm{i}} \dot{W}^{\prime \prime \prime \prime}+C_{\mathrm{e}} \dot{W}-\bar{E} h \eta_{0} W^{\prime \prime} \\
& \quad-\frac{\bar{E} h}{2 l}\left[\int_{0}^{l} W^{\prime 2}(\xi) \mathrm{d} \xi\right] W^{\prime \prime}-P(X, t)=0 .
\end{aligned}
$$

The nondimensional variables $x, w$ and $\tau$ defined for the undamaged panel are also used to nondimensionalize equation (5). One obtains

$$
\begin{aligned}
S_{\mathrm{r}} \cdot & w^{\prime \prime \prime \prime}+\ddot{w}+C_{\mathrm{I}} \dot{w}^{\prime \prime \prime \prime}+C_{\mathrm{E}} \dot{w}-S_{\mathrm{r}} \cdot R_{x} w^{\prime \prime} \\
& -S_{\mathrm{r}} \cdot S\left[\int_{0}^{1} w^{\prime 2}(\xi) \mathrm{d} \xi\right] w^{\prime \prime}-F(x, \tau)=0,
\end{aligned}
$$

where $S_{\mathrm{r}}$ is defined as the stiffness reduction factor, $S_{\mathrm{r}}=\bar{D} / D$, characterizing the damage level in the panel, i.e. $S_{\mathrm{r}}$ equals unity when no damage is present. Also, the smaller the value of $S_{\mathrm{r}}$, the larger the damage.

Finally, the panel dynamics is computed by using a finite difference scheme and a time marching algorithm. All spatial derivatives in the governing partial differential equation and the boundary conditions are approximated by a finite difference formulation of second-order accuracy. The discretized equations for the dynamics are integrated in time using the Gear method, which is adequate for stiff systems. More details regarding the numerical approach may be found in Epureanu et al [7].

\section{Nonlinear feedback excitation}

Damage detection in strongly nonlinear systems is challenging especially because of the modal coupling and the amplitude dependence of the response to harmonic excitation. Thus, current methods are designed to avoid or minimize the influence of nonlinearities and, hence, have low sensitivity. The proposed solution for greatly increasing this sensitivity is to exploit (and enhance) nonlinearities. The key idea is to actively change the dynamics so that the attractor (in state space or in embedded coordinates) is a complex geometric shape which is used as a feature able to indicate the damage level and location. Next, given a (measured) attractor, damage is detected by comparing its current shape with its undamaged shape.

In the case of limit cycle oscillations, the attractors are closed curves in the state space. Such curves are good indicators in some cases [11], but they are not very sensitive [7, 8] or rich because their shape is not very complex. To increase sensitivity, the geometric complexity is enhanced by inducing chaotic dynamics which usually has attractors expanded spatially (and of similar magnitude). These attractors have complex shapes which are sensitive to damage. A very large increase in sensitivity may be obtained by using a nonlinear feedback excitation. The specific form of the nonlinear feedback is dependent on each particular application and includes quadratic, cubic and possibly other terms. This novel approach is significantly different from a nonlinear or chaotic excitation. A chaotic excitation applied to a linear system means that superposition still holds and, hence, each frequency component of the chaotic signal is simply filtered through the transfer function of the structure as any other type of excitation would be. However, when using nonlinear feedback (superposition does not hold), the excitation becomes part of the system. The emerging dynamics is a characteristic of this new system and, thus, it is much more sensitive to changes in its parameters. Moreover, the present method detects the damage location and extent with increased performance. In contrast, chaotic excitation has been used for damage detection with some success $[14,26]$, but the features used there (e.g. attractor variance, Lyapunov exponents) could not predict the damage location and were only moderately sensitive to the damage level.

To show the potential offered by nonlinear feedback excitation for enhancing the sensitivity of damage detection to the level, the extent and the location of the damage in the panel, a concentrated force in the form of a harmonic excitation in combination with nonlinear feedback force components is used and applied to the middle point of the panel. The response function of nonlinear feedback excitation may be expressed as

$$
F(\tau)=F_{0} \sin \omega \tau+\lambda w(1 / 2)^{2} w^{\prime \prime}(1 / 2)+\gamma w^{\prime \prime}(1 / 2),
$$

where $w(1 / 2)$ and $w^{\prime \prime}(1 / 2)$ are the displacement and the curvature at the middle point of the panel, and $\lambda$ and $\gamma$ are control parameters. The form of the nonlinear components in feedback excitation is drawn from the form of two terms in equation (4), $S\left[\int_{0}^{1} w^{\prime 2}(\xi) \mathrm{d} \xi\right] w^{\prime \prime}$ and $R_{x} w^{\prime \prime}$, corresponding to structural nonlinearity and axial pre-load, which play an important role in the complex dynamics of the panel. Thus, nonlinear feedback excitation may be designed to increase the nonlinearity and enhance the instability in the linear panel without considering structural nonlinearity and axial preload. Finally, the transverse distributed loading $F(x, \tau)$ in equation (4) can be expressed as $F(\tau) \delta(x-1 / 2)$, where $F(\tau)$ is the input force and $\delta$ is the Dirac delta function.

As known for linear dynamics, when a linear system is subjected to a harmonic excitation, the ensuing dynamics is a periodic oscillation. Such an oscillation/vibration has been observed in the literature to have a low sensitivity to parametric variations $[8,9]$. For example, in the case of a linear panel model excited by a concentrated harmonic force acting on the middle point of the panel, the attractor of the dynamics (of the quarter point of the panel) is just a simple elliptical curve. 
(a) No Damage

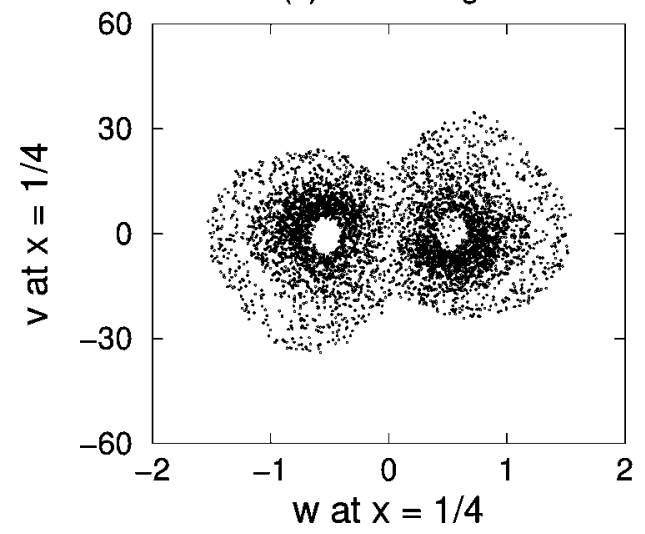

(b) High Level; Small Extent



Figure 3. The Poincaré plot of the attractor of the dynamics spanned by the displacement and velocity of the quarter point for undamaged and damaged panels subjected to nonlinear feedback excitation at their middle points.

Figure 2 shows such an elliptical curve for three separate cases: a healthy panel and two panels with low and high levels of damage expanding over a small extent. One may note that it is difficult to distinguish between the dynamics of these three systems by only observing the vibration of the quarter point on the panel. Here, the linear panel model can be obtained by eliminating the nonlinear term $S\left[\int_{0}^{1} w^{\prime 2}(\xi) \mathrm{d} \xi\right] w^{\prime \prime}$ from the nonlinear panel model in equation (6) (discussed in section 2). The low level and small extent of damage in figure 2 are considered to be a $10 \%$ loss of bending stiffness of the panel (corresponding a value of 0.9 for $S_{\mathrm{r}}$ in equation (6)) expanding along $1.25 \%$ of the panel length around the middle point of the panel. Also, a 50\% loss of bending stiffness of the panel is referred to as a high level of damage.

In contrast, the dynamics of the panel model forced by nonlinear feedback excitation is much more sensitive to parametric changes. The shape of an attractor changes when parameters of the system change [8]. Such changes have been observed [26], but their characterization has been limited. To address this issue, one considers that the attractor (sampled in time) is a cloud of points in state space or embedded coordinates. This cloud of points moves and deforms when parametric changes occur. To characterize these shape changes, algorithms (such as the ones applied for the recognition of human faces [12]) may be used. In essence, the novel idea is to collect clouds of points corresponding to healthy and damaged systems (with various levels and locations of damage). Then, changes in the shape and orientation of the clouds can be monitored and correlated quantitatively and/or qualitatively with parametric variations. Such an approach allows for the detection of multiple simultaneous damages of various levels and locations. Distinct from pattern recognition applied to other signals, the proposed approach uses attractors (invariants of the dynamics) which are much more intimately/better tied to system parameters.

Figure 3 shows that the geometric shape of a cloud of points (in a chaotic attractor) changes dramatically when damage is present. That is an important improvement in sensitivity over the linear approaches where exactly the same damage levels and extents cannot be detected (as shown in figure 2). Thus, the nonlinearity in the linear panel generated by nonlinear feedback excitation can effectively generate a more complex geometric shape for the attractor of the dynamics, and can also increase significantly the sensitivity of the shape of the attractor to damage presence, location and extent.

To characterize the changes in the shape of the attractors we define and use probability density functions which represent the probability for a given point in the attractor to be in a vicinity (of a given size) of a given point, i.e. the number of points in the attractor which are inside a circle of radius $r$ around each point in the state space, divided by the total number of points in the attractor. For a $2 \mathrm{D}$ cloud of points, a three-dimensional diagram of probability densities is obtained as shown in figure 4. The radius $r$ is chosen as 0.1 . The particular value for $r$ is dependent on the size and the number of points in the attractor.

Figure 4 shows the probability density function of the attractors of the dynamics for an undamaged panel and a panel with a low level and a high level of damage (for a small extent of damage) located respectively at the middle point and at the quarter point of the panel length. Figure 5 shows that the difference between the probability density functions for a healthy panel and the panels with different locations and levels of damage is large. Moreover, the shape of the probability differences differs for various types and locations of damage. The parametric variations considered represent different levels, extents and locations of damage. As a result, the nonlinear feedback excitation can successfully enhance nonlinearity in the system to provide an accurate and robust method for detecting the level, extent and location of structural damage.

Next, we investigate in more detail the detection of the level and location of damage. Figure 6 shows the difference probability density functions obtained for three separate cases of a panel with different levels of damage around its middle point (for values of $S_{\mathrm{r}}$ of $0.50,0.55$ and 0.60 ). The three shapes observed are very similar. To quantify their similarity, one may compute a two-point correlation matrix and calculate its eigenvalues. As expected, one of the eigenvalues obtained, $e_{1}^{m}$, is much larger than the other two, i.e. $e_{2}^{m} / e_{1}^{m}=0.0283$ and $e_{2}^{m} / e_{1}^{m}=0.0144$. The clear separation of one of the eigenvalues compared to the other two indicates that the three shapes observed in figure 6 are linearly dependent. 


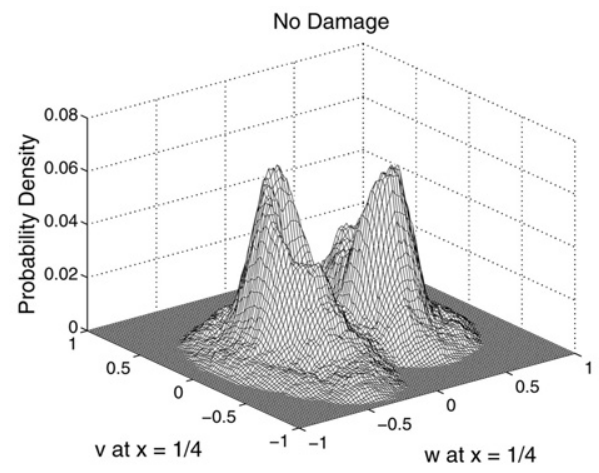

Small damage at the middle point



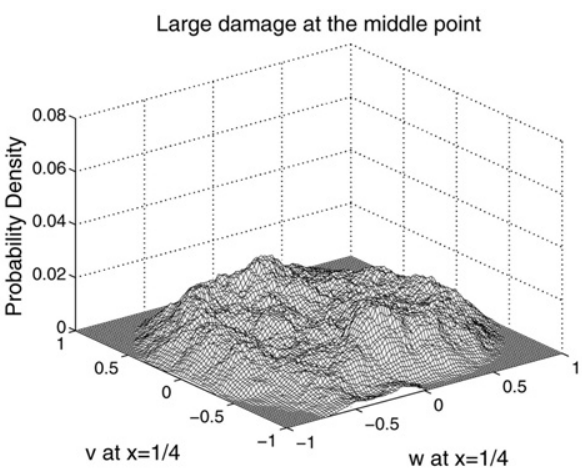

Small damage at the quarter point

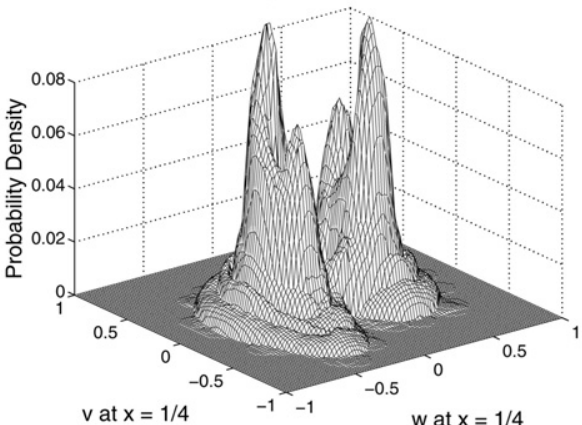

Figure 4. The probability density of the point distribution in the Poincare sections for the undamaged panel and the panel with low and high levels (and small extent) of damage at different locations.


Figure 5. The difference of probability densities of the distribution of points in the Poincare plot for the panel with different locations and levels of damage.
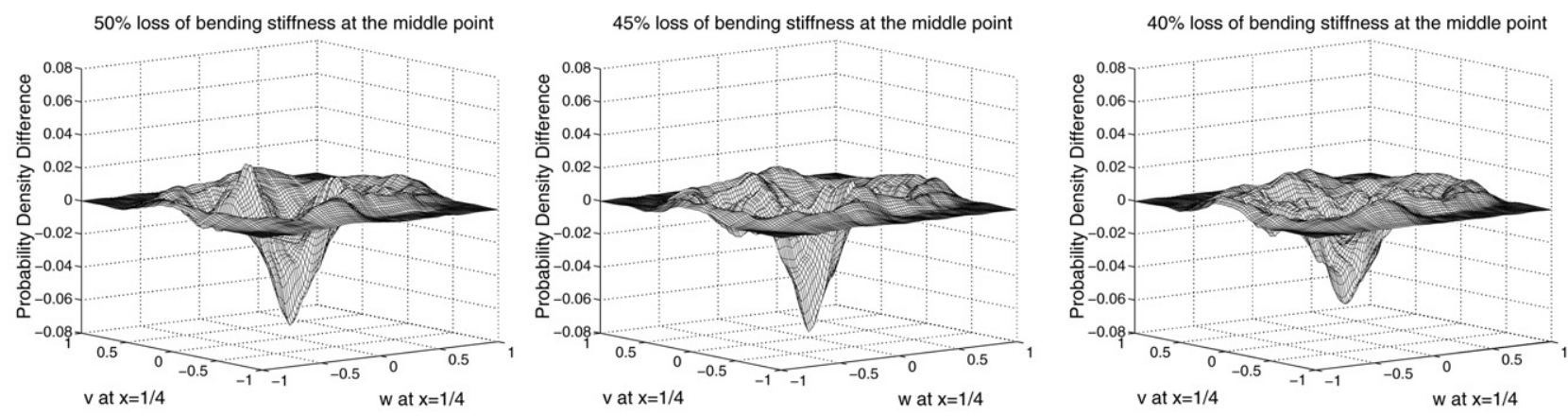

Figure 6. The difference of probability densities of the distribution of points in the Poincare plot for the panel with different levels of damage around its middle point (left $S_{\mathrm{r}}=0.50$, center $S_{\mathrm{r}}=0.55$, right $S_{\mathrm{r}}=0.60$ ).

The most dominant shape corresponds to $e_{1}^{m}$ and is denoted by $\mathbf{s}_{1}^{m}$. Similarly, figure 7 shows the difference probability density functions obtained for panels with different levels of damage around the quarter point (for values of $S_{\mathrm{r}}$ of 0.50 ,
0.55 and 0.60 ). The same separation of the eigenvalues of the two-point correlation matrix is obtained. Specifically, one obtains $e_{2}^{q} / e_{1}^{q}=0.0480$ and $e_{2}^{q} / e_{1}^{q}=0.0229$. The most dominant shape corresponds to $e_{1}^{q}$ and is denoted by 



Figure 7. The difference of probability densities of the distribution of points in the Poincaré plot for the panel with different levels of damage around its quarter point (left $S_{\mathrm{r}}=0.50$, center $S_{\mathrm{r}}=0.55$, right $\left.S_{\mathrm{r}}=0.60\right)$.


Figure 8. The difference of probability densities of the distribution of points in the Poincare plot for the panel with different levels of simultaneous damages around its quarter and middle points (left $S_{\mathrm{r}}=0.50$, center $S_{\mathrm{r}}=0.55$, right $S_{\mathrm{r}}=0.60$ for both damage locations).

$\mathbf{s}_{1}^{q}$. These results demonstrate that the difference probability density functions have approximately the same shape (but of varying magnitude) for various damage levels and that this shape changes when the damage location changes.

Similarly, figure 8 shows the difference probability density functions obtained for distinct panels with simultaneous damages of different levels at two locations: around the middle point and around the quarter point (for values of $S_{\mathrm{r}}$ of $0.50,0.55$ and 0.60 at both locations). The difference probability density functions are linearly dependent also, as demonstrated by the eigenvalues of a two-point correlation matrix, $e_{2}^{m q} / e_{1}^{m q}=$ 0.0409 and $e_{2}^{m q} / e_{1}^{m q}=0.0253$. However, the dominant shape, $\mathbf{s}_{1}^{m q}$ (corresponding to $e_{1}^{m q}$ ), is linearly dependent on the shapes $\mathbf{s}_{1}^{m}$ and $\mathbf{s}_{1}^{q}$ obtained for damage present only at the middle point and, separately, damage only at the quarter point. This linear dependence is shown by the eigenvalues of a two-point correlation matrix computed using nine shapes (three for middle point damage only, three for quarter point damage only and three for simultaneous damages) plotted in figure 9. The log plot indicates that there is a large separation between the first two eigenvalues and the rest of the seven. The presence of two dominant eigenvalues indicates that the nine shapes are approximately linearly dependent on only two shapes, which correspond to plates with damage at the quarter point and separately at the middle point.

\section{Conclusions}

The dynamics of a panel forced by transverse loads and undergoing limit cycle oscillations and chaos has been investigated. The nonlinear von Karman plate theory has

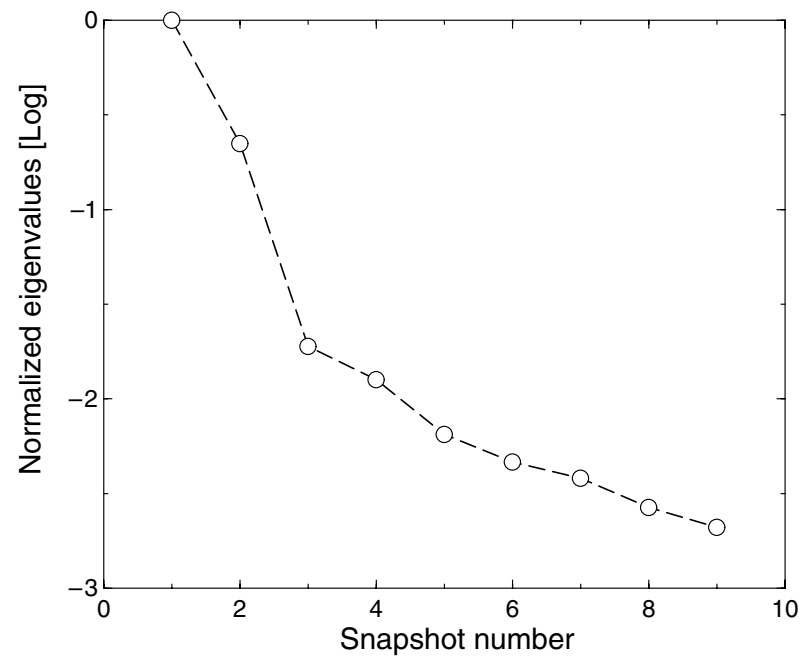

Figure 9. A log plot of the eigenvalues of the correlation matrix for nine snapshots obtained as the difference probability density functions for nine separate damage cases at two locations (all eigenvalues are divided by the largest eigenvalue).

been used to obtain a model for the panel coupled with a nonlinear feedback excitation. Damage has been modeled as a loss of stiffness in a portion of the plate. The sensitivity of the nonlinear system to parametric changes has been shown to be an effective tool in detecting damages. Most of the current studies of such problems are based on linear theories. In contrast, the results presented have been obtained using nonlinear dynamics, and have the advantage of an increased sensitivity and accuracy in detecting damage. 


\section{Acknowledgments}

The authors wish to acknowledge the National Science Foundation (CAREER program) and Professor Masayoshi Tomizuka and Dr Shih-Chi Liu (program directors) for the generous support of this work.

\section{References}

[1] Abdalla M O, Grigoriadis K M and Zimmerman D C 1998 Enhanced structural damage detection using alternating projection methods AIAA J. 36 1305-11

[2] Agbabian M S, Masri S F, Miller R F and Caughey T K 1990 System identification approach to detection of structural changes ASCE J. Eng. Mech. 117 370-90

[3] Amaravadi V K, Mitchell K, Rao V S and Derriso M M 2002 Structural integrity monitoring of composite patch repairs using wavelet analysis and neural networks Proc. SPIE: Smart Structures and Materials 2002: Smart Structures and Integrated Systems (San Diego, CA) vol 4701, ed L P Davies (Bellingham, WA: SPIE Optical Engineering Press) pp 156-66

[4] Atalla M J and Inman D J 1998 On model updating using neural networks Mech. Syst. Signal Process. 12 135-61

[5] Chatterjee A, Cusumano J P and Chelidze D 2002 Optimal tracking of parameter drift in a chaotic system: experiment and theory J. Sound Vib. 250 877-901

[6] Doebling S W, Farrar C R, Prime M B and Shevitz D W 1996 Damage identification and health monitoring of structural and mechanical systems from changes in their vibration characteristics: a literature review Report LA-13070-MS Los Alamos National Laboratories, Los Alamos, NM

[7] Epureanu B I, Tang L S and Paidoussis M P 2004 Coherent structures and their influence on the dynamics of aeroelastic panels Int. J. Non-Linear Mech. 36 977-91

[8] Epureanu B I, Tang L S and Paidoussis M P 2004 Exploiting chaotic dynamics for detecting parametric variations in aeroelastic systems AIAA J. 42 728-35

[9] Farrar C R, Doebling S W and Nix D A 2001 Vibration-based structural damage identification Phil. Trans. R. Soc. A 359 $131-49$

[10] Ghanem R and Shinozuka M 1995 Structural system identification: theory ASCE J. Eng. Mech. 121 255-64

[11] Kesaraju R V and Noah S T 1994 Characterization and detection of parameter variations of nonlinear mechanical systems Nonlinear Dyn. $6433-57$

[12] Kirby M and Sirovich L 1990 Application of the Karhunen-Loève procedure for the characterization of human faces IEEE Trans. Pattern Anal. Mach. Intell. 12 103-8

[13] Ljung L 1999 System Identification-Theory for the User (New York: Prentice-Hall)
[14] Logan D and Mathew J 1996 Using the correlation dimension for vibration fault diagnosis of rolling element bearings: basic concepts Mech. Syst. Signal Process. 10 241-50

[15] Loh C H and Tou I C 1995 A system identification approach to the detection of changes in both linear and nonlinear structural parameters Earthq. Eng. Struct. Dyn. 24 85-97

[16] Pandey A K and Biswas M 1994 Damage detection in structures using changes in flexibility J. Sound Vib. 169 3-17

[17] Pappa R P, James G H and Zimmerman D C 1998 Autonomous modal identification of the space shuttle tail rudder J. Spacecr. Rockets 35 163-9

[18] Pecora L M and Caroll T L 1996 Discontinuous and nondifferentiable functions and dimension increase induced by filtering chaotic data Chaos 6 432-9

[19] Sadeghi M H and Fassois S D 1997 Geometric approach to nondestructive identification of faults in stochastic structural systems AIAA J. 35 700-5

[20] Smyth A W, Masri S F, Caughey T K and Hunter N F 2000 Surveillance of mechanical systems on the basis of vibration signature analysis J. Appl. Mech. 67 540-51

[21] Smyth A W, Masri S F, Chassiakos A G and Caughey T K 1999 On-line parametric identification of MDOF nonlinear hysteretic systems ASCE J. Eng. Mech. 125 133-42

[22] Sohn H and Farrar C R 2001 Damage diagnosis using time series analysis of vibration signals Smart Mater. Struct. 10 446-51

[23] Tumer I Y, Wood K L and Busch-Vishniac I J 2000 Monitoring of signals from manufacturing processes using the Karhunen-Loève transform Mech. Syst. Signal Process. 14 1011-26

[24] van Overschee P and DeMoor B 1996 Subspace Identification for Linear Systems: Theory, Implementation and Applications (New York: Kluwer)

[25] Wang W J, Wu Z T and Chen J 2001 Fault identification in rotating machinery using the correlation dimension and bispectra Nonlinear Dyn. 25 383-93

[26] Yang B, Suh C S and Chan A K 2002 Characterization and detection of crack-induced rotary instability J. Vib. Acoust. 124 40-8

[27] Zang C and Imregun M 2001 Structural damage detection using artificial neural networks and measured FRF data reduced via principal component projection J. Sound Vib. 242 813-27

[28] Zimmerman D C 1999 Looking into the crystal ball: the continued need for multiple viewpoints in damage detection Key Eng. Mater. Damage Assessment Struct. 167/168 76-90

[29] Zimmerman D C 2000 Model validation and verification of large and complex space structures Inverse Problems Eng. 8 93-118

[30] Zimmerman D C, Yap K and Hasselman T 1999 Evolutionary approach for model refinement Mech. Syst. Signal Process. 13 609-25 